\title{
Brownian Dynamics Simulation for Fully-Developed Flow of Dilute Polymer Solution through a Parallel-Plate Channel
}

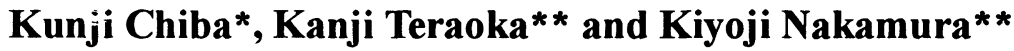 \\ * Faculty of Education, Shiga Universiy, 2-5-1 Hiratsu, Otsu, Shiga, 520-0862 Japan \\ ** Department of Mechanophysics Engineering, Graduate School of Engineering, Osaka University, \\ 2-1 Yamada-Oka, Suita, Osaka, 565-0871 Japan
}

Received 15 March 1999; received in revised form 25 May 1999

\begin{abstract}
The effect of the Weissenberg number on a fully-developed flow of dilute polymer solution is numerically examined using Brownian dynamics simulation. The results demonstrate the ability of a mesoscopic approach to predict accurate results in a viscoelastic flow problem using a kinetic theory model: a FENE dumbbell model.
\end{abstract}

Keywords: Brownian dynamics simulation; FENE dumbbell; Dilute polymer solution; Fullydeveloped flow

\section{Introduction}

To date, most numerical simulations of viscoelastic flows have been based on a purely macroscopic approach where one solves numerically the conservation laws together with a suitable constitutive equation. Since the pioneering work by Laso and Öttinger ${ }^{[1,2]}$, i.e. the CONNFFESSIT approach, a complementary mesoscopic approach to viscoelastic flow simulations is now emerging. This approach combines the solution of the conservation laws with the direct use of a kinetic theory model, such as FENE (finitely extensible nonlinear elastic) dumbbell or chain model, describing the fluid rheology ${ }^{[3-6]}$.

For illustrative purposes, in the present study we try to compute a fully-developed flow of dilute polymer solution through a parallel-plate channel using the Brownian dynamics simulation. A mechanism of the change in velocity and stress profiles caused by existence of molecules in polymer solution flows will be then discussed on the basis of microscopic structure: distribution of alignment and extension of molecules. The polymer solution is described by the kinetic theory of FENE dumbbells.

\section{Governing equations}

For FENE dumbbells ${ }^{[7]}$, the spring force is defined as

$$
\boldsymbol{F}^{(c)}=\frac{H Q}{1-\left(Q / Q_{0}\right)^{2}} \quad\left(Q \leq Q_{0}\right)
$$

where $Q$ is the connector vector (vector connecting the two beads of the dumbbell), $Q$ is the length of the connector vector, $Q_{0}$ is the maximum allowable extension of the FENE spring and $H$ is the spring constant.

The velocity field is assumed to be the form of $u=u(y)$, $\nu=0$ which automatically satisfies the continuity equation for an incompressible fluid, where $u$ and $v$ are $x$-(flow-direction) and $y$-(width-direction) components, respectively. The velocity profile can then be calculated from the momentum balance equation:

$$
-\frac{\partial p}{\partial x}+\eta_{s} \frac{\partial^{2} u}{\partial y^{2}}+\frac{\partial \tau_{x y}}{\partial y}=0
$$

where $p$ is the isotropic pressure, $\eta_{s}$ is the solvent viscosity and $\tau_{x y}$ is the contribution of the polymer to the shear stress, which is obtained from a simulation of polymer dynamics. It is possible to estimate the stress from a stochastic simulation of a suitable ensemble of FENE dumbbells. The time-discretized dynamics of each individual FENE dumbbell can be expressed in the following form:

$$
Q(t+\Delta t)=Q(t)+\left\{\kappa \cdot Q(t)-\frac{2 H / \zeta}{1-\left(Q(t) / Q_{0}\right)^{2}} Q(t)\right\} \Delta t+G(\Delta t)
$$

$$
\langle G(\Delta t)\rangle=0,\langle G(\Delta t) G(\Delta t)\rangle=\frac{4 k_{B} T \Delta t}{\zeta} I
$$

\footnotetext{
* Corresponding author Fax:+81-77-537-7840, E-mail: kchiba@sue.shiga-u.ac.jp
} 
where $\kappa$ is the velocity gradient tensor, $\zeta$ is the friction coefficient of a bead, $G$ is the random displacement of the bead due to Brownian motion which has zero mean and a variance expressed by eq.(4), $k_{B}$ is the Boltzmann's constant, $T$ is the absolute temperature, $\Delta t$ is the time step and $I$ is the unit tensor.

A large number of dumbbells are simulated according to eqs. (3), (4) and the polymer contribution to the stress tensor $\tau$ is computed as an ensemble average using the Kramers form.

$$
\tau=n H\left\langle\frac{Q Q}{1-\left(Q / Q_{0}\right)^{2}}\right\rangle-n k_{B} T I,
$$

where $n$ is the number density of dumbbells,.

In the computations all variables are normalized using the half width of the channel $L$, the mean fluid velocity $U_{0}$ and $Q_{0}$ in the following form:

$$
\begin{aligned}
& x=L x^{*}, \quad y=L y^{*}, \quad \boldsymbol{u}=U_{0} \boldsymbol{u}^{*}, \quad p=n k_{B} T p^{*}, \tau=n k_{B} T \tau^{*}, \\
& \kappa=\left(U_{0} / L\right) \kappa^{*}, \quad \eta_{s}=n k_{B} T\left(L / U_{0}\right) \eta_{s}^{*}, \quad Q=Q_{0} Q^{*}, \\
& \boldsymbol{G}=Q_{0} \boldsymbol{G}^{*}, \quad t=\left(L / U_{0}\right) t^{*}, \\
& b=\frac{H Q_{0}^{2}}{k_{B} T}, \quad \lambda_{H}=\frac{\zeta}{4 H}, W e=\frac{\lambda_{H}}{L / U_{0}},
\end{aligned}
$$

$b$ is referred to as the FENE parameter, $\lambda_{H}$ is the relaxation time and $W e$ is the Weissenberg number. A symbol of * expressing the dimensionless quantities is omitted hereinafter. Then the stochastic equation of the dumbbell

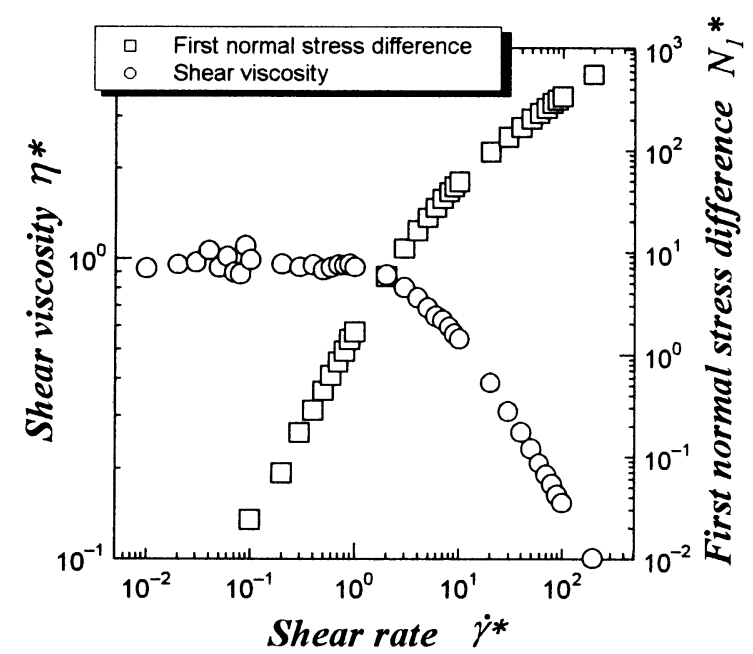

Fig.1 Brownian dynamics simulation of the shear viscosity and the first normal stress difference of the FENE dumbbells for $b=100$. dynamics (eqs.(3), (4)) can be rewritten as

$$
\begin{gathered}
Q(t+\Delta t)=Q(t)+\left\{\kappa \cdot Q(t)-\frac{Q(t)}{2 W e\left(1-Q^{2}(t)\right)}\right\} \Delta t+\boldsymbol{G}(\Delta t) \\
\langle\boldsymbol{G}(\Delta t)\rangle=0,\langle\boldsymbol{G}(\Delta t) \boldsymbol{G}(\Delta t)\rangle=\frac{\Delta t}{b W e} \boldsymbol{I}
\end{gathered}
$$

\section{Numerical simulation procedure}

In the computations the flow rate was adjusted to unity by changing the pressure drop $\partial p / \partial x$. The boundary condition on a channel wall was a no-slip condition.

The solution scheme is outlined as follows:

(1) The velocity field for Newtonian flow is given first.

(2) The connector vector $\boldsymbol{Q}$ of each individual FENE dumbbell is computed from the equilibrium condition of $Q$ using the stochastic equation (7), (8) at all mesh points in the given velocity field until the steady state of $Q$ can be obtained. The time step is $\Delta t=0.01$, mesh size is $1 / 20$ and the number of dumbbells in an ensemble of FENE dumbbells is 200 .

(3) The polymer contribution to the stress tensor are estimated as the time-averaged $\tau$ during $5000 \Delta t$ in the steady state.

(4) Eq.(2) is recomputed with the newly estimated extra stress to obtain the new value of $u$.

This procedure from step (2) to (4) is repeated until convergence is obtained in the predicted velocity and stress fields.

In the present study we examined the effects of the Weissenberg number, the FENE parameter and the solvent

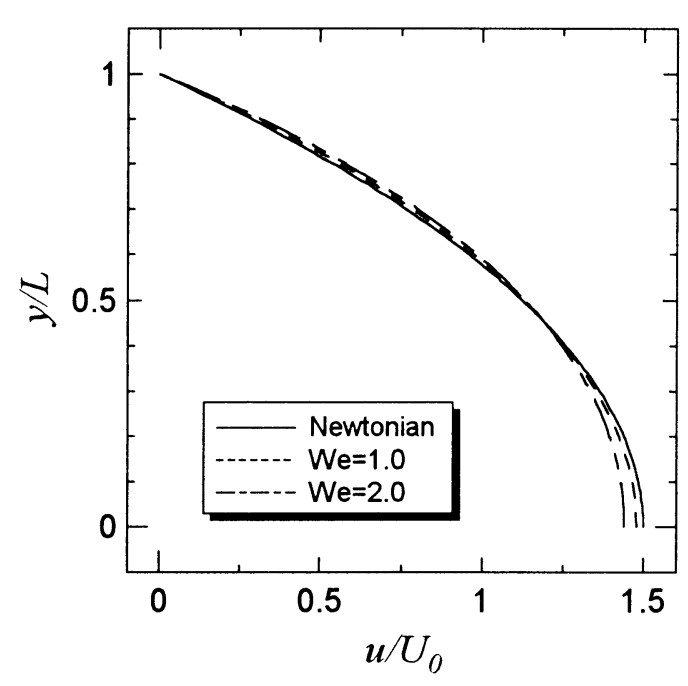

Fig.2 Velocity profile along the channel width for $b=100$, $\eta_{s}{ }^{*}=0.1$. 


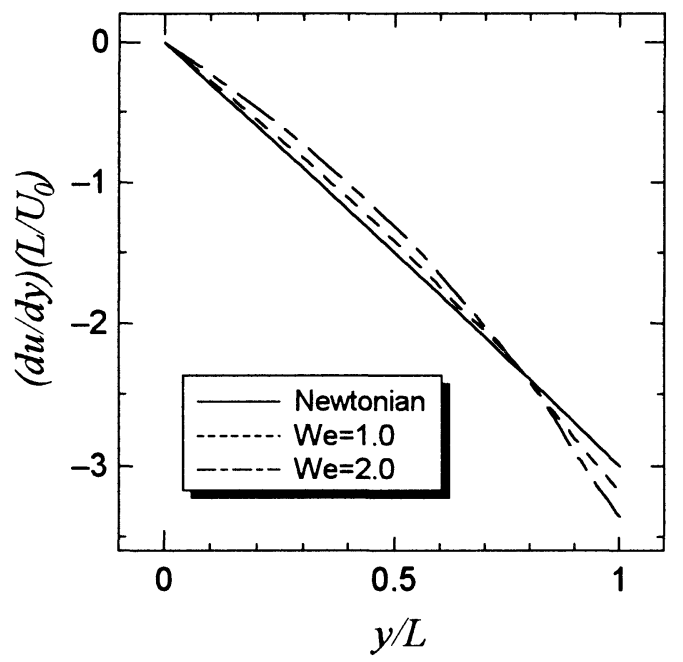

(a)

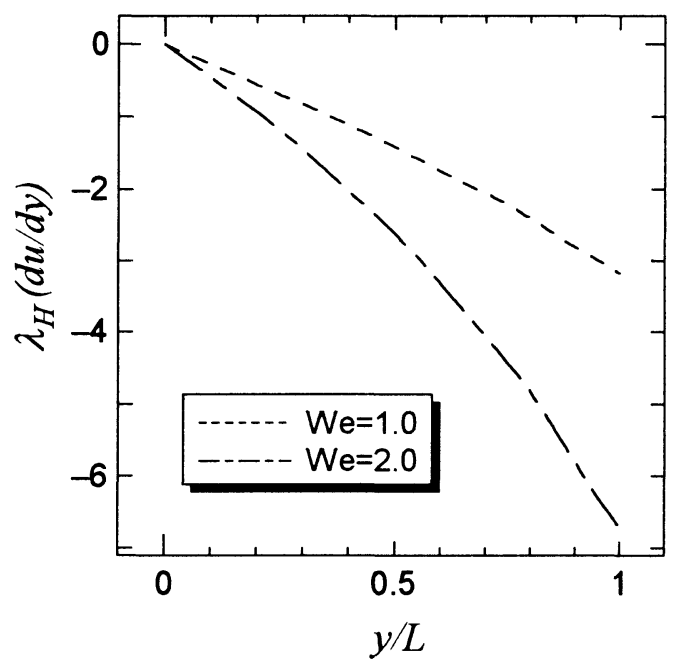

(b)

Fig.3 Velocity gradient profile along the channel width for $b=100, \eta_{s}{ }^{*}=0.1$; (a) dimensionless velocity gradient with $L / U_{0}$; (b) dimensionless velocity gradient with $\lambda_{H}$.

viscosity on the flow field. The only results for $W e=1$ and 2, $b=100, \eta_{s}=0.1$ are shown here for the sake of brevity. Flow rates corresponding to $W e=1$ and 2 are 1.002 and 0.997 at $\partial p / \partial x=-2.85$ and -4.59 , respectively.

\section{Results}

The results of the polymer contribution to the shear viscosity $\eta^{*}$ and the first normal stress difference $N_{1} *$ for $b=100$ are shown in Fig.1. The shear viscosity, the first normal stress difference and the shear rate are normalized as $\eta^{*}=\left(\eta-\eta_{s}\right) / n k_{B} T \lambda_{H}, \quad N_{1}^{*}=N_{1} / n k_{B} T$ and $\dot{\gamma} * \lambda_{H} \dot{\gamma}$ in the figure. The solution of the FENE dumbbells exhibits not only shear-thinning viscosity but also large and positive first normal stress difference with a dependence on shear rate that

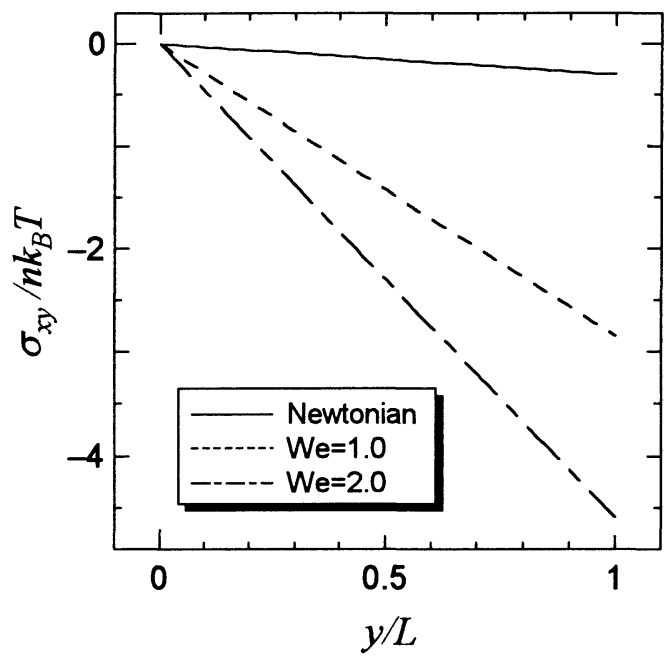

(a)

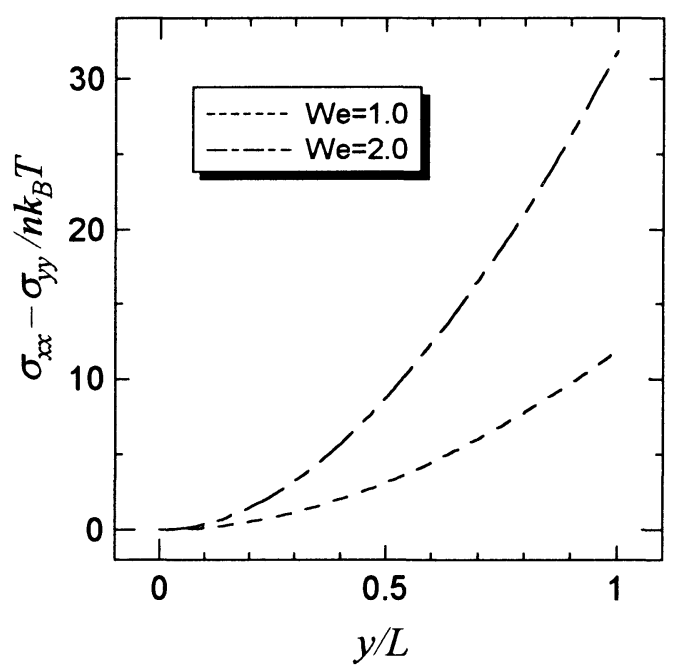

(b)

Fig.4 Stress distribution along the channel width for $b=100$, $\eta_{s^{*}}=0.1$; (a) shear stress; (b) the first normal stress difference.

is weaker than quadratic. This tendency is consistent with the rheological properties of polymer solutions. Details of the rheological behavior can be obtained in the previous papers $^{[8,9]}$

The effect of the Weissenberg number on the velocity and stress fields, and the orientation state of dumbbells is described now. The velocity profiles for the FENE dumbbell solutions become more plug-like profile as the Weissenberg number is increased in Fig.2: flatter velocity profile in the region around the centerline of the channel can be predicted. This is due to shear thinning behavior of the solution and the reduction of shear viscosity near the channel wall. Figure 3 (a),(b) shows the variation of the dimensionless velocity gradient along the channel width. The quantity $\lambda_{H} \partial u / \partial y=$ $\partial u / \partial y\left(L / U_{0}\right) W e$ is the dimensionless shear rate used in Fig.1, which can be calculated from the values of 
$(\partial u / \partial y)\left(L / U_{0}\right)$ in Fig.3(a). The range of shear rate for $W e=1$ and 2 in the predicted fully-developed flow are $\lambda_{H} \dot{\gamma}=0 \sim-3$ and $0 \sim-7$, respectively. The tendency shown in Fig.3(b) is caused by both an increase in the mean velocity $U_{0}$ and a decrease in channel width $L$ as the Weissenberg number is increased.

Furthermore, Figs.4(a),(b) and 5 show that the shear stress, the first normal stress difference and the dumbbell length increase as the channel wall is approached and the Weissenberg number is increased, i.e. as the shear rate is further increased. The linear trend of the shear stress of the FENE dumbbell solutions can also be observed similarly to that for Newtonian flow. The orientation state of dumbbells can be illustrated concisely using an orientation ellipsoid, and the projections on the $x y, y z$ and $z x$-planes of the orientation ellipsoid are shown in Fig.6. Because of the same reason above, the dumbbell orientation and dumbbell alignment in the flow direction become improved as the channel wall is approached and the Weissenberg number is increased.

\section{Conclusions}

A mesoscopic approach can clearly show that alignment of molecules in the flow direction becomes better as the channel wall is approached in a fully-developed flow of polymer solutions; shear viscosity is further decreased. A plug-like flow occurs owing to the mechanism above in polymer solution flow. The above results demonstrate the ability of Brownian dynamics simulation to produce accurate results in a viscoelastic flow problem using a kinetic theory model for the polymer dynamics, such as a FENE dumbbell. The next step is to apply the mesoscopic approach to complex flow problems of polymer solutions.

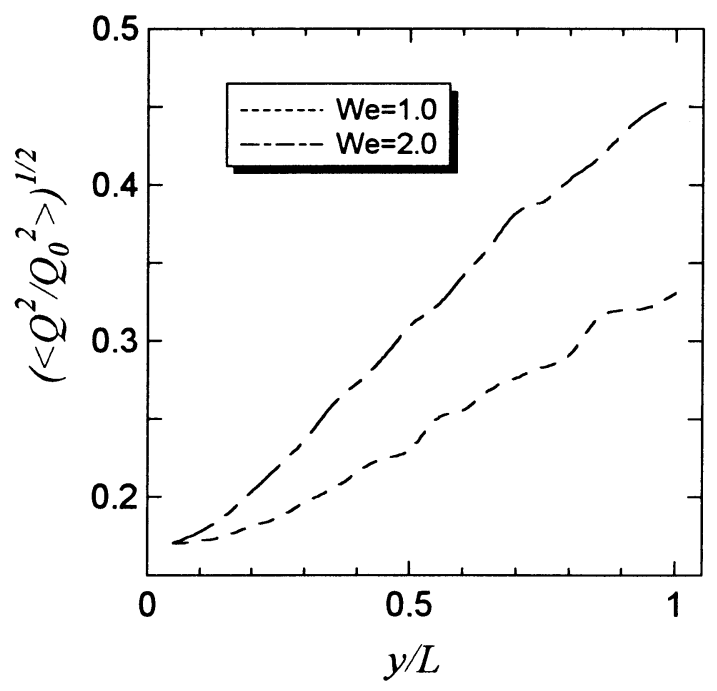

Fig.5 Profile of the root-mean-square of dumbbell length along the channel width for $b=100, \eta_{s}{ }^{*}=0.1$.
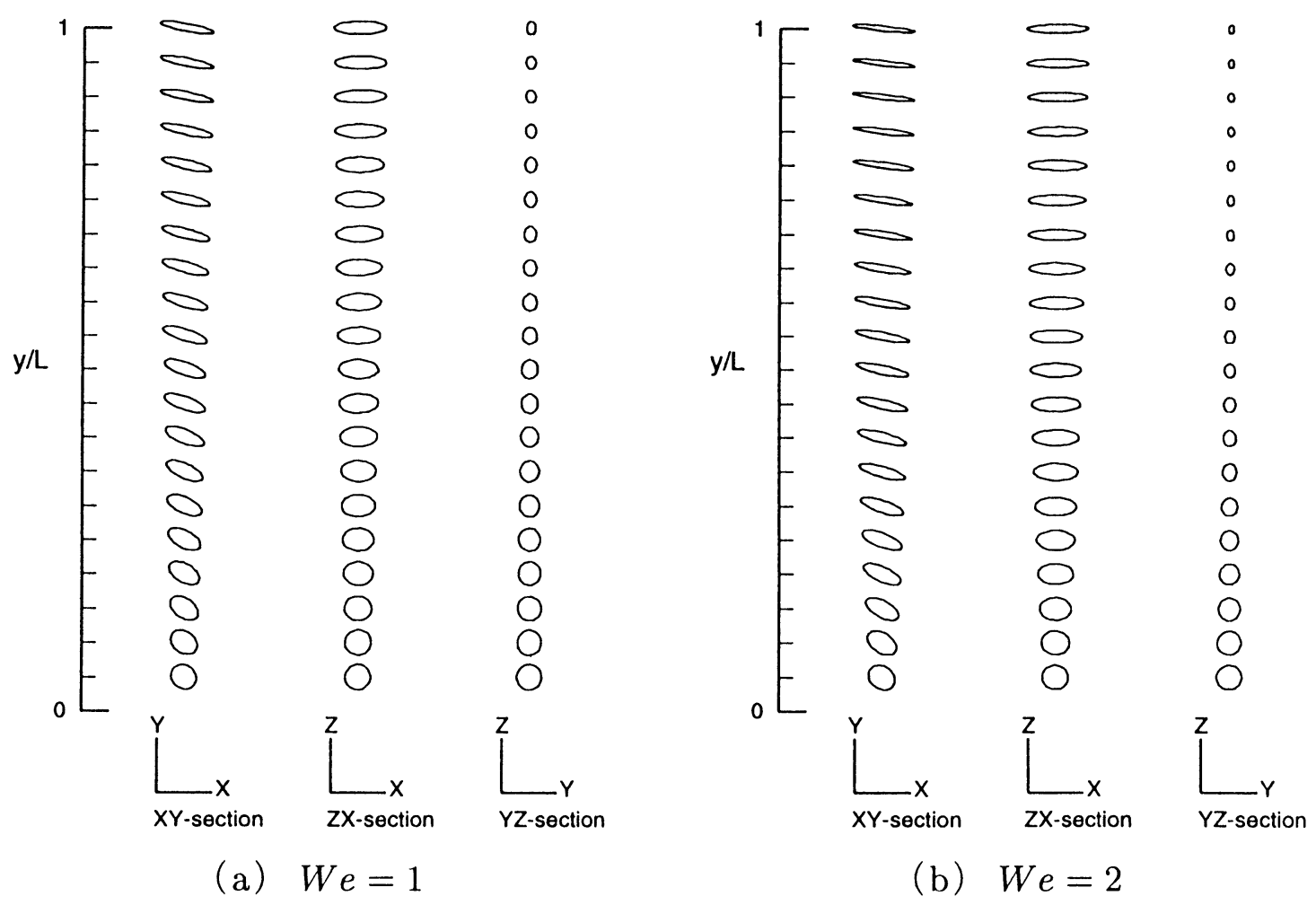

Fig.6 Orientation ellipsoid of dumbbells along the channel width for $b=100, \eta_{s}{ }^{*}=0.1$; (a) $W e=1$; (b) $W e=2$. 


\section{References}

[1] H.C. Öttinger, M. Laso, "Theoretical and Applied Rheology", edited by P. Moldenaers and R. Keunings, p.286-288, Elsevier, Amsterdam (1992)

[2] M. Laso, H.C. Öttinger, J. Non-Newtonian Fluid Mech., 47, 1(1993)

[3] K. Feigl, M. Laso, H.C. Öttinger, Macromolecules, 28, 3261(1995)
[4] M.A. Hulsen, A.P.G. van Heel, B.H.A.A. van den Brule, J. Non-Newtonian Fluid Mech., 70, 79(1997)

[5] C.C. Hua, J.D. Schieber, J. Rheol., 42, 477(1998)

[6] A.P.G. van Heel, M.A. Hulsen, B.H.A.A. van den Brule, J. Non-Newtonian Fluid Mech., 75, 253(1998)

[7] H.R. Warner, Ind. Eng. Chem. Fundam., 11, 379(1972)

[8] K. Chiba, K. Teraoka, Y. Iokito, K. Nakamura, NIHON REOROJI GAKKAISHI, 27, 31(1999)

[9] K. Chiba, K. Teraoka, Y. Iokito, K. Nakamura, NIHON REOROJI GAKKAISHI, 27 (1999) in press 